\title{
KnoWare: A System for Citizen-based Environmental Monitoring
}

\author{
Jeremy Storer, Joseph Chao, Andrew Torelli, and \\ Alexis Ostrowski \\ Bowling Green State University Bowling Green, Ohio, USA
}

\author{
jstorer@bgsu.edu; jchao@bgsu.edu; torelli@bgsu.edu; \\ alexiso@bgsu.edu
}

\begin{abstract}
Non-expert scientists are frequently involved in research requiring data acquisition over large geographic areas. Despite mutual benefits for such "citizen science", barriers also exist, including 1) difficulty maintaining user engagement with timely feedback, and 2) the challenge of providing non-experts with the means to generate reliable data. We have developed a system that addresses these barriers. Our technologies, KnoWare and InSpector, allow users to: collect reliable scientific measurements, map geo-tagged data, and intuitively visualize the results in real-time. KnoWare comprises a web portal and an iOS app with two core functions. First, users can generate scientific 'queries' that entail a call for information posed to a crowd with customized options for participant responses and viewing data. Second, users can respond to queries with their GPSenabled mobile device, which results in their geo- and time-stamped responses populating a webaccessible map in real time. KnoWare can also interface with additional applications to diversify the types of data that can be reported. We demonstrate this capability with a second iOS app called InSpector that performs quantitative water quality measurements. When used in combination, these technologies create a workflow to facilitate the collection, sharing and interpretation of scientific data by non-expert scientists.
\end{abstract}

Keywords: Citizen Science, Cell-phone Spectrometer, Water Contaminant Analysis, Web and Mobile Development, Visualization

\section{Introduction}

The prevalence of smartphones represents an opportunity to gather, create, and consume data from individuals on a scale that has not previously been available. Even the 'average' mobile devices in widespread use have significant computing power and are typically GPS-enabled. Scien-

Material published as part of this publication, either on-line or in print, is copyrighted by the Informing Science Institute. Permission to make digital or paper copy of part or all of these works for personal or classroom use is granted without fee provided that the copies are not made or distributed for profit or commercial advantage AND that copies 1) bear this notice in full and 2) give the full citation on the first page. It is permissible to abstract these works so long as credit is given. To copy in all other cases or to republish or to post on a server or to redistribute to lists requires specific permission and payment of a fee. Contact Publisher@InformingScience.org to request redistribution permission. tific research that requires widespread sampling over geographic areas can benefit greatly by mobilizing members of the population equipped with these devices to conduct scientifically useful measurements or activities. Data generated in this way can complement existing data collection methods. One key advantage of these 'citizen science' initiatives is that sourcing large groups of individuals is an efficient strategy to 
collect large-scale data sets because contributors typically volunteer their time in exchange for meaningful participation. However, in spite of the benefits, multiple barriers work against increased prevalence of citizen science initiatives, including 1) the difficulty maintaining prolonged user engagement with meaningful feedback, and 2) the challenge of providing non-experts with the means to collect reliable data.

We have developed technologies to directly address these barriers by providing 'citizen scientists' with 1) an easy-to-use mechanism to participate in scientific investigations with real time visual feedback, and 2) software and a device that interface with iPhones to collect reliable scientific measurements from color-based test kits in widespread use. Our technologies currently focus on allowing users to record water quality measurements since such data collection requires large, repeated measurement, and there are many existing citizen science initiatives already focused on water quality that may benefit. Our technology platform, called KnoWare, comprises a paired web portal and iOS application for data visualization and mapping. In this report, we use KnoWare in combination with an additional iOS application we created (InSpector) to process images taken with the iPhone camera using an add-on device. The InSpector app allows users to obtain reliable readings of colorimetric water quality tests that currently depend on subjective color-comparisons. These technologies work together to provide an engaging workflow for the collection and interpretation of environmental water quality data by non-professional, citizen scientists.

\section{Literature Review}

The technologies we have developed facilitate citizen science based data collection efforts with the following motivations: increasing exposure and 'literacy' in STEM areas, the potential impact of involving the broader public in scientific efforts to advance research, and opportunities to engage students at our own institution in the development of software and devices with 'real-world' applications. In this research, we focus on citizen science based water quality monitoring, partially because of the shutdown of the water supply for approximately 500,000 people for 3 days in August, 2014, when a toxin-producing algal bloom contaminated the water supply in our local area (Fitzsimmons, 2014). In fact, such algal blooms are a recurring issue in Lake Erie (Cheung, Liang, \& Lee, 2013; Michalak et al., 2013), and have captured significant attention in our local region (Cha \& Stow, 2015). Since this water supply shutdown, there has been interest in the region for citizens to participate in water quality monitoring (Henry, 2014).

In addition, global access to clean water suitable for human consumption and use is a growing concern worldwide, especially in light of threats to both water quality and availability (Gleeson, Wada, Bierkens, \& van Beek, 2012; Gleick, 1993; Vörösmarty, Green, Salisbury, \& Lammers, 2000; Vörösmarty et al., 2010). One of the main threats to water quality is eutrophication, which entails the release of nutrients into water supplies that fuel excessive algae and plant growth (Heisler et al., 2008; Withers, Neal, Jarvie, \& Doody, 2014). Such algal blooms pose multiple problems including production of hazardous toxins, reductions in species diversity, aquatic oxygen depletion and reduction of harvestable fish and shellfish (Smith \& Schindler, 2009). For example, a 2009 report released by the United Nations stated:

"Globally, the most prevalent water quality problem is eutrophication, a result of high-nutrient loads (mainly phosphorus and nitrogen), which substantially impairs beneficial uses of water... Major nutrient sources include agricultural runoff, domestic sewage (also a source of microbial pollution), industrial effluents and atmospheric inputs from fossil fuel burning and bush fires." (World Water Assessment Programme, 2009) pg. 138

While different sources of eutrophication have been identified, additional research is needed to understand how the increased nutrients in waterways interact with other factors in the environ- 
ment and contribute to decreased water quality (Smith \& Schindler, 2009; Withers et al., 2014). Therefore, there is widespread interest in monitoring the levels of key nutrients in environmental water supplies.

Our goal is to facilitate water quality efforts by deploying technologies that foster citizen involvement, increase understanding about water quality, and provide new sources of scientificallymeaningful data. Improving access to citizen science initiatives has the potential to expand awareness and interest in pertinent issues such as water quality and clean water access by the general public.

Involving the broader public in scientific research efforts has been applied in numerous settings for considerably longer than when the term 'citizen science' was coined two decades ago (Gura, 2013). Given the need to address environmental concerns such as eutrophication with multifaceted management strategies, citizen science offers additional benefits. For example, participation in citizen science initiatives is an effective approach to involve stakeholders and the public in ecosystem management(Conrad \& Hilchey, 2011; Pollock \& Whitelaw, 2005). Furthermore, the process and outcomes of environmental decision making has been shown to benefit from increased involvement of stakeholders (Beierle, 2002). To ensure the quality of our water resources in the future, these citizen science efforts are important since it is human behavior changes that are critical to sustainable preservation of water quality (Fischer et al., 2012; Pahl-Wostl, 2002; Vlek \& Steg, 2007).

Citizen science programs that are aimed at educating and involving citizens in water conservation efforts are well positioned to undertake large-scale water quality measurements. As discussed previously however, there is a need to maintain consistent participation from the engaged user base and to ensure reliable collection of the water quality measurements by non-professional scientists.

\section{Overview of the Technology Workflow}

KnoWare is a system that provides users with the ability to create, respond to, and visualize data from scientific queries in real-time. KnoWare is intended for use in investigations where mapping the location of user-responses is important, a requirement that can be easily accomplished using GPS-enabled mobile devices. For example, many social science, political science, ecological and environmental investigations depend on geospatial mapping of data. In our pilot application for water quality monitoring, an example query might be "What is the concentration of nitrate present in the water at your location?" To respond to this query, we envisioned multiple participants at various locations in a region who would report results from simple water contaminant tests already in widespread use. While in the field, users would operate with a workflow depicted in Figure 1. First, the user would assemble a foldable InSpector device, which enables the smartphone camera to take images of colored water contaminant test solutions. These images would then be analyzed with an easy-to-use InSpector app to calculate concentrations of contaminants based on whichever test kit they are using. The results are then mapped along with the user's GPS location (as reported by the user's smartphone) for visualization via a mobile app or desktop web portal. The full dataset can also be downloaded for optional post-processing. This relatively simple and straightforward approach will facilitate participation in citizen-science initiatives by providing tools to quickly analyze water quality in a water sample, report findings, and view collective results in real time. 


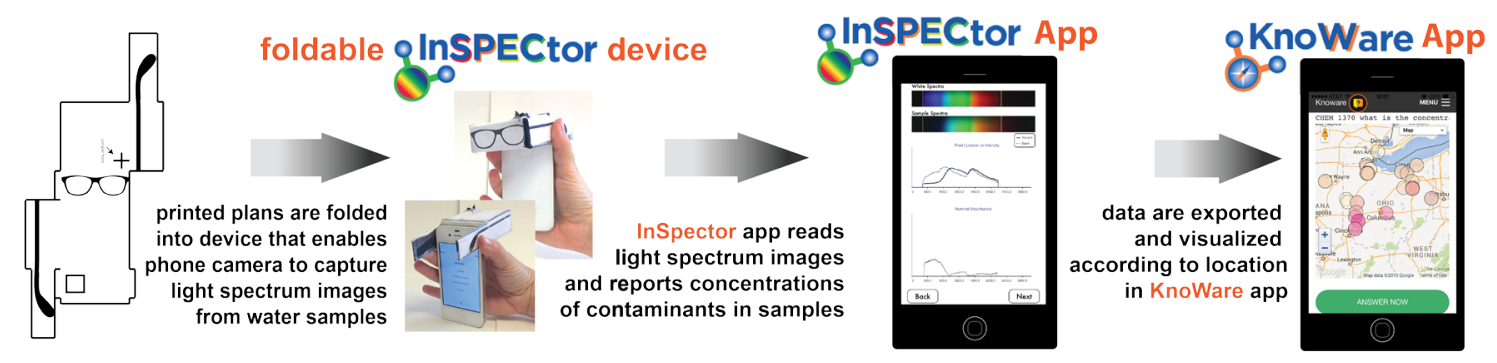

Figure 1: The KnoWare technology workflow to measure and report water quality data.

The following sections will describe in more detail the combination of technologies underlying the workflow we have described.

\section{KnoWare Web Portal and Mobile app}

At the core of the technology workflow is the KnoWare web portal and mobile app that collectively serve two important functions. The KnoWare web portal is designed for users to create what we refer to as a query, essentially a question that will be posed to a crowd in order to collect scientifically- and geographically-relevant responses. The KnoWare web portal also acts as a place for users to visualize responses to a query or to export a table of the response data to use with third-party visualization software and analysis if desired. Finally, the KnoWare mobile app allows participants to respond to a query with data that are time- and geo-stamped. These functions are further described below.

The first function of KnoWare is to help users create custom queries, which is performed on a dedicated web page in the KnoWare web portal (Figure 2). The required inputs from the user mainly include text for the actual question of interest that will be posed to the crowd, constraints on the type and range of allowed responses, an optional link to a website with supplemental instructions intended for query participants, and the option to allow participants to attach an image to their submitted response. Defining the allowed response types is critical since this will change how the results are displayed visually. Thus, when creating a query, a user must first consider what types of responses will be recorded. KnoWare is currently limited to queries with multiplechoice, numerical (both defined increment or continuous scale), or yes/no binary response types.

Once a response type is selected, the user can choose how pins on the web map will be colored to aid in visualization and interpretation of the data. How the colors themselves are selected is designed to be flexible to allow responses for any query to be represented by a meaningful set of colors (Figure 2B, inset).

For example, consider a query designed to map responses to the question "What is the concentration of nitrate present in the water at your location?" where participants were expected to perform sample measurements in the field using a simple dipstick test kit. The colors for the query can be customized to match that of the desired test kit to facilitate both participant responses by matching the on-screen colors for user response options and the resulting data accordingly (Figure 3). The color picker within KnoWare (Figure 2B black inset) is adaptable for a range of response types and color schemes, including discrete color choices or custom color gradients that are automatically filled in after the user selects two boundary colors (Figure 2B). 

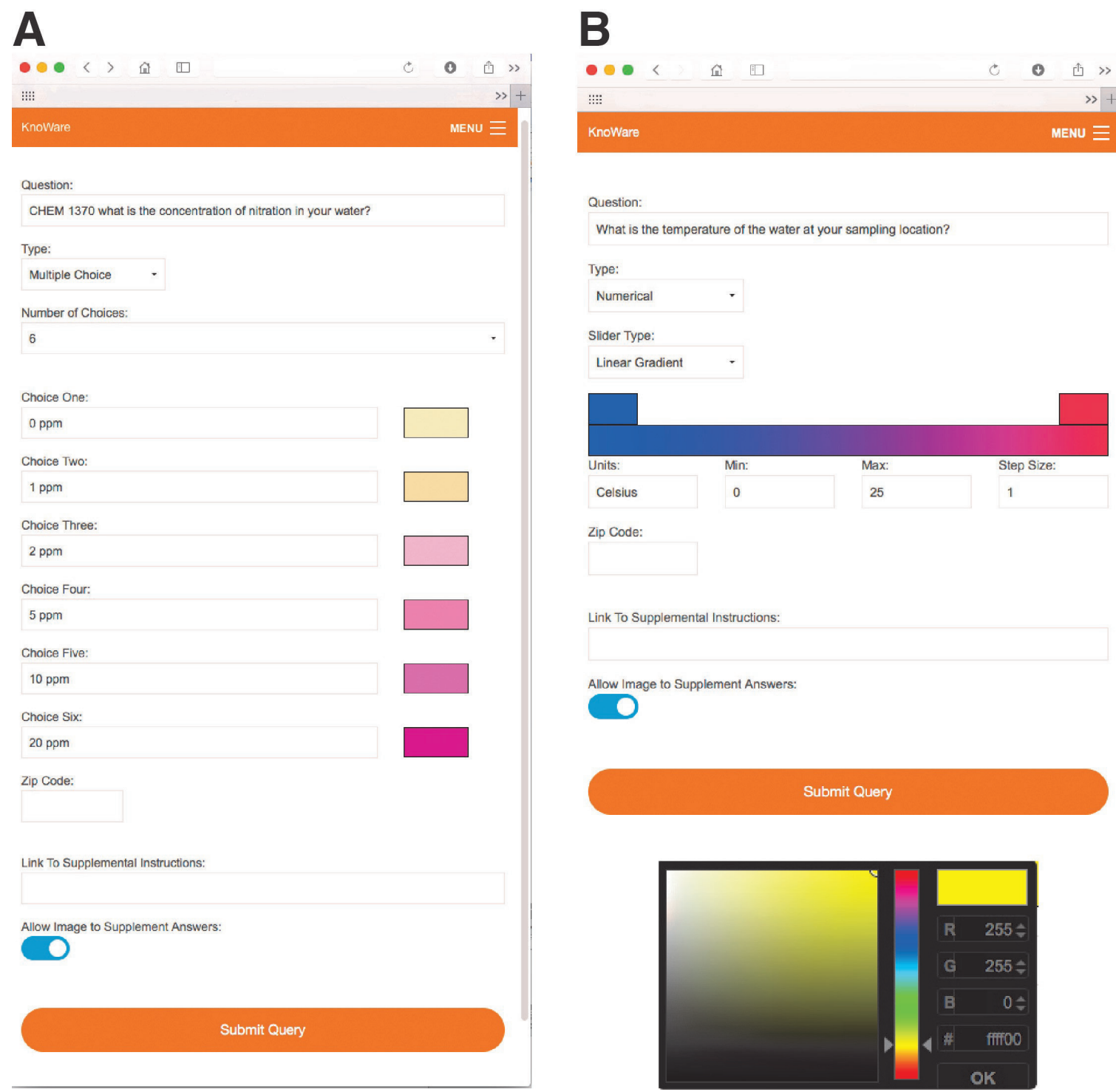

Figure 2: A: Creating a multiple choice query with 3 discrete answers that each has a unique user-defined color selected.

B: Creating a numerical-response query with a custom color gradient that can be chosen for meaningful correspondence with answers. The black inset shows the custom color picker popup feature.

With the coloring assigned, the user is then able to submit the query to the database where it is assigned a unique identification code. This identification code can be used to share the query with a crowd of potential participants. For example, a teacher could create a query and distribute the unique identification code to students in a course so they are able to participate in a joint investigation.

Once the query is created, it will appear in the web portal in a list of available queries for participants to respond to. All responses are submitted via the mobile application at the site where participants are gathering data in the field so GPS location can be automatically recorded. A participant is also able to browse through the list of available queries or enter a query identification code. Once a query is selected, a map is shown with color-coded pins representing the data submitted up to that point. Using the KnoWare mobile app, participants can contribute their response 
to a selected query, which is uploaded to the web portal along with a timestamp, the longitude and latitude of the participant's location, and an optional image taken with the mobile device camera.
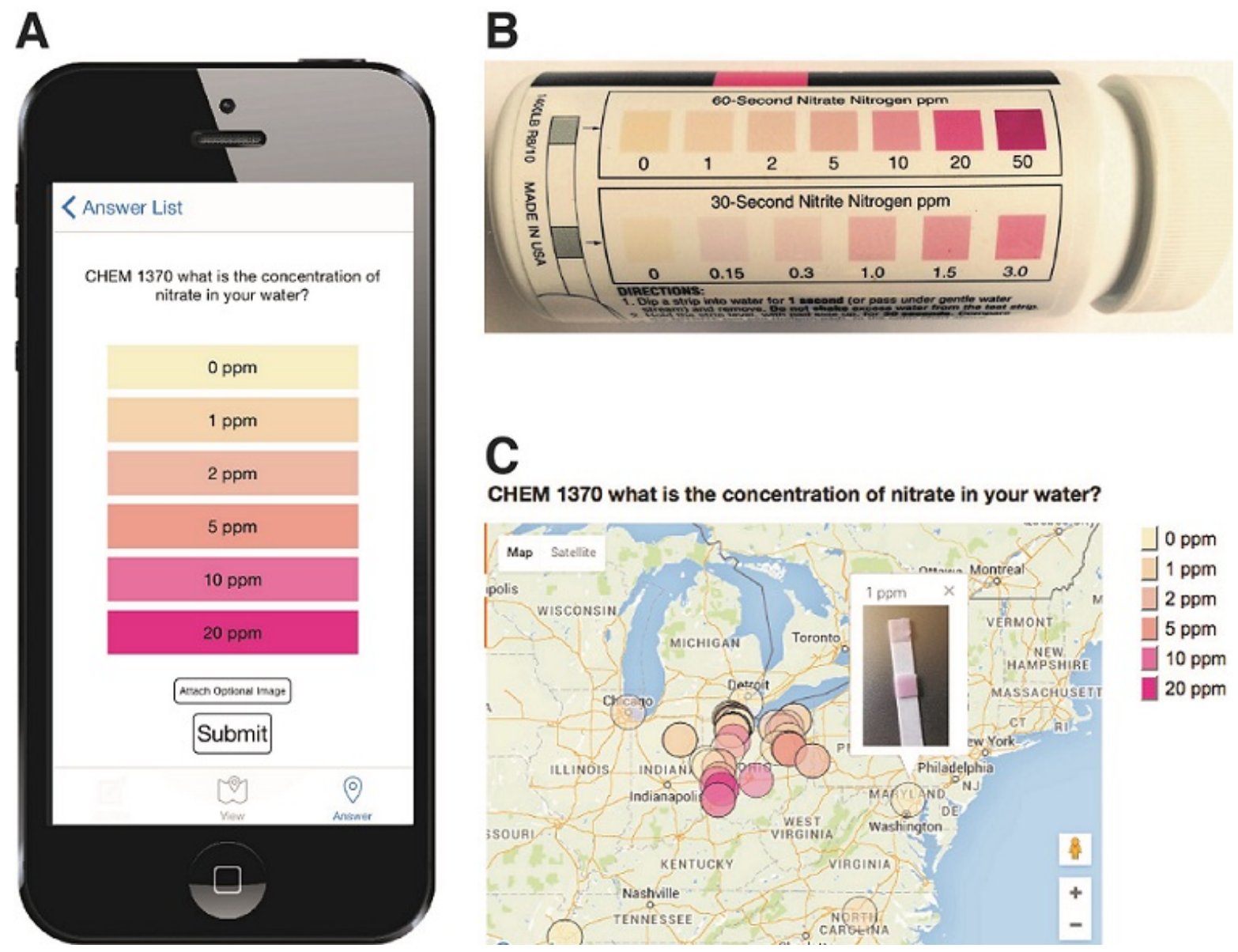

Figure 3: Custom colors in KnoWare aid in user responses and data visualization.

A.) Each multiple choice response color has been chosen to match possible results obtained from a commercially available nitrate test kit shown in $B$.

C.) Pins created on the results map from a participant response is also colored according to the test kit result and will display a popup inset image if the optional image is attached by the user to their response.

Large numbers of responses can quickly become overwhelming. An important goal of the KnoWare web portal is therefore to incorporate intuitive visualization options to assist with data interpretation. The results map for each query displays semi-transparent circles representing the locations of reported measurements (Figure 3C). Each circle is color-coded according to the magnitude of the reported measurement (in this case they correspond to the specific colors of the test kit from Figure 3B ranging from beige to pink). The color-coding conveys immediately understandable information to the viewer. Other visualizations are in development to further expand options for data visualization using KnoWare. If images were submitted by participants with their responses, these can be viewed by clicking on given pin (Figure 3C inset). If desired, third-party software can be used for visualization or analysis by downloading the dataset in comma-separated values format (.csv) via a link on every query response page. 


\section{Light-based Water Contaminant Analysis}

The KnoWare web portal and mobile application primarily function to solicit user responses to scientific queries and visualize the results. In order to diversify the types of data that can be reported, KnoWare can also accept the output from other apps. For the purposes of our pilot into water quality testing, we needed a way for users to be able to measure water contaminant information with improved reliability. In this case, multiple parameters are of interest, including $\mathrm{pH}$ or the concentration of various nutrients such as phosphorous and nitrate. A variety of inexpensive, commercially-available water quality testing kits are currently used by non-professional scientists to measure various water quality parameters, and they frequently rely on the user to match the color of a solution to a cue-card in order to estimate contaminant concentration (Figure 4). For example, to determine the concentration of nitrate in a water sample, users must add a test reagent solution into their water sample, which changes color according to how much nitrate is present in the water sample. The user then estimates the nitrate concentration by matching the color of the solution to the included standard card. Our strategy was to employ an established approach to quantifying the color of solutions, which we will describe here briefly before introducing the app and device we developed for this purpose.

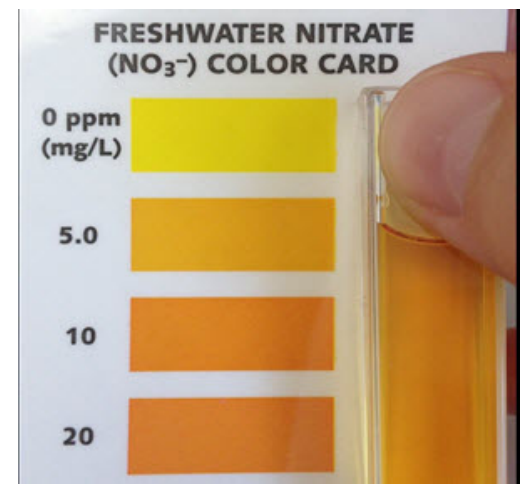

Figure 4: Estimating the concentration of nitrate in a water sample requires users to match colors with a color card, which is prone to judgment error.

When illuminated by white light (e.g., sunlight), colored solutions appear as they do because their contents selectively absorb certain colors of the spectrum, in effect changing the combination of light colors that reach our eyes. For example, solutions that appear orange contain compounds that selectively absorb blue light. In other words, the spectrum of light after passing through the sample is depleted of blue light, such that the light transmitted through the sample that reaches our eye is dominated by the red, orange, and yellow colors of light. Measuring the wavelengths (i.e., color) of light absorbed by a sample is a widely employed strategy for the quantitative analysis of colored solutions. Figure 5 illustrates this approach wherein a device (in this case a prism) is used to generate a spectrum from a light source. The intensity of light at each wavelength is recorded for this 'raw light' spectrum. When a colored solution is placed in the beam path of light, the intensity of light transmitted through the sample at certain wavelengths will diminish in a concentration-dependent manner. 

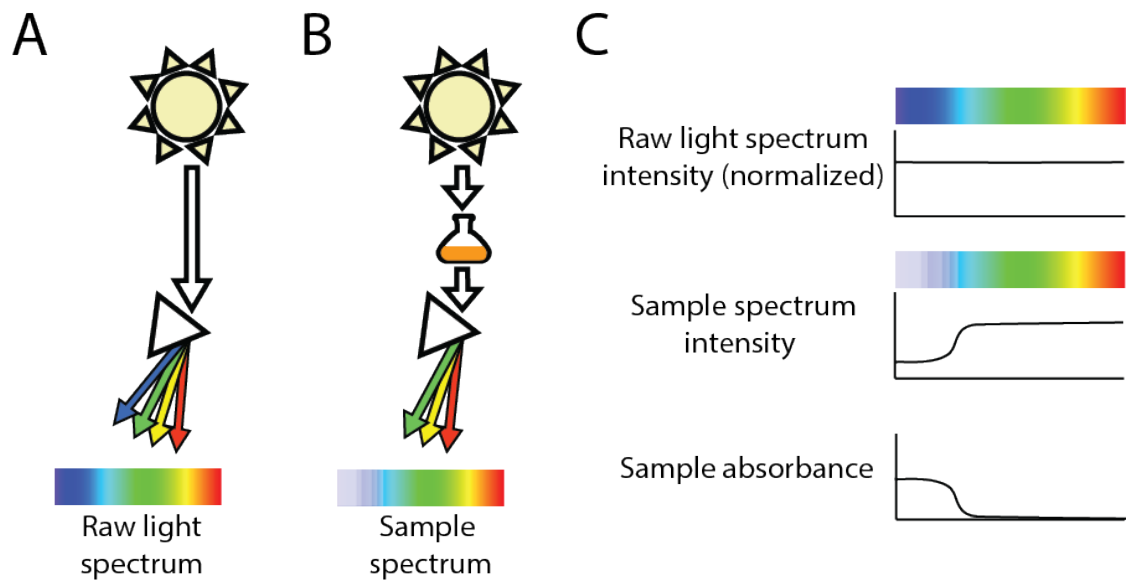

Figure 5.

A. White light passing through a prism (triangles) is spread into a spectrum of its component colors (raw light spectrum).

B. The same white light shining through a sample that appears colored due to absorbance of certain colors from the white light source, in this case violet and blue light.

C. Plot of the normalized intensity of the raw white light spectrum (upper plot) and intensity of light after passing through colored sample (middle plot). Comparison of the raw and sample light intensities are conventionally represented as an absorbance plot to quantify the specific colors of light absorbed by the sample (lower plot).

Thus, comparing the intensity of light in the "raw" light and "sample" spectra can be achieved by comparing the intensity of light from two "slices" through each spectrum. It is standard to report this comparison by plotting the absorbance for all wavelengths, or light colors, in the spectrum according to:

$$
\text { Absorbance }(\text { at light wavelength } \lambda)=\log _{10}\left(\frac{\text { Intensity of raw light at wavelength } \lambda}{\text { Intensity of light transmitted through sample at wavelength } \lambda}\right)
$$

In order to accurately determine the concentration of a contaminant in a sample solution, the absorbance profiles are first plotted for a series of solutions with known contaminant concentrations using the appropriate colorimetric test kit. The absorbance profile from samples with unknown contaminant concentrations can then be compared with this standard plot to accurately estimate the concentration of the contaminant.

\section{InSpector Device}

In order to pursue the strategy for water contaminant analysis described above, we created a foldable cardboard device for use with a mobile-phone camera (Figure 6). The device uses diffraction grating instead of a prism to spread the light spectrum so the intensities of its component colors can be measured separately. This is a well-established methodology, and we were further inspired for our device design by the work of others who have previously reported on foldable spectroscope devices for use with mobile phones (Public Lab Staff, 2015; Scheeline \& Kelley, 2009; Science Buddies Staff, 2015; Wakabayashi \& Hamada, 2006). Our device has a sample test-tube port. This allows for us to make these absorbance measurements, a key difference in our device from some others. 

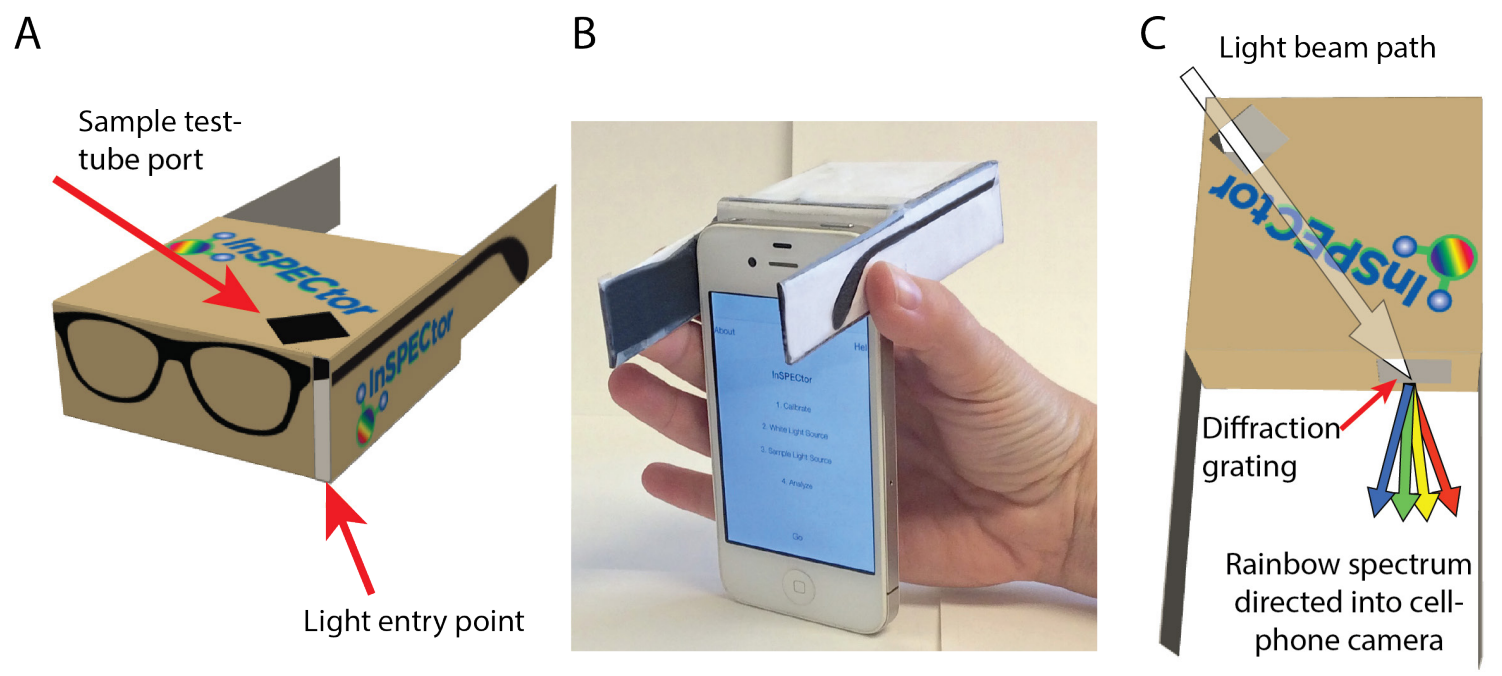

Figure 6: InSpector device.
A. CAD rendering of the InSpector device highlighting the cutout for inserting sample test tubes and the sunlight entry point.
B. View of InSpector device used with an iPhone.
C. Light beam path through the InSpector device including location of diffraction grat- ing that directs rainbow spectrum into cell-phone camera.

\section{InSpector - An iOS Application to Analyze Light Spectra}

The InSpector device enables users to take snapshots of raw light and 'sample' light spectra, respectively. In order to process these images and to arrive at an estimate of contaminant concentration, we created an additional iOS app we are calling InSpector. The InSpector application is designed to eliminate the need for color matching by eye to estimate contaminant concentrations using many popular water test kits. By providing a direct measurement, the objective of the InSpector app is to improve the accuracy and reproducibility of water quality measurements.

The InSpector app analyzes the contaminant concentrations by translating images of rainbow spectra taken with the InSpector device into absorbance spectra. The application then compares the absorbance value from an environmental sample against a calibration curve for a known water contaminant to interpolate the contaminant concentration in the unknown sample. The user can select which test kit they are using from a pre-programmed list and the sample absorbance will be compared with the appropriate pre-loaded standard curve. Measuring sample absorbance in this way is powerful because, in theory, it can be applied to any test that involves solutions that change color due to the presence of some compound (as long as the corresponding standard curve has been determined). InSpector also has a built in feature to port the measured contaminant concentration to the KnoWare app as part of a response to a relevant query.

To use InSpector, a user first attaches the InSpector device to their iPhone (Figure 6). Then a 'raw' light spectrum is captured, typically from sunlight, but other sources of broadband light such as incandescent bulbs can be used as well (Figure 7A, 7B). To measure the sample light spectrum, a colored solution is put into a test tube that is inserted into the sample port on the InSpector device (Figure 6) and illuminated with the same light source. Figure 7C shows the raw and sample light spectra with a plot of their respective intensities measured across a horizontal slice through each spectrum. InSpector converts these two intensity plots into a plot of nominal absorbance (Figure 7D), which is then compared with a standard curve generated ahead of time 
for the particular test kit being used. Finally, the data (contaminant concentrations) are displayed onscreen and may be ported to KnoWare for mapping.

The InSpector application is built natively in the iOS environment to ensure optimal run speed and responsiveness. Native design also allows important control of camera parameters including ISO and shutter speed, for which values were optimized for best performance with the InSpector device and kept constant between the raw and sample spectra.
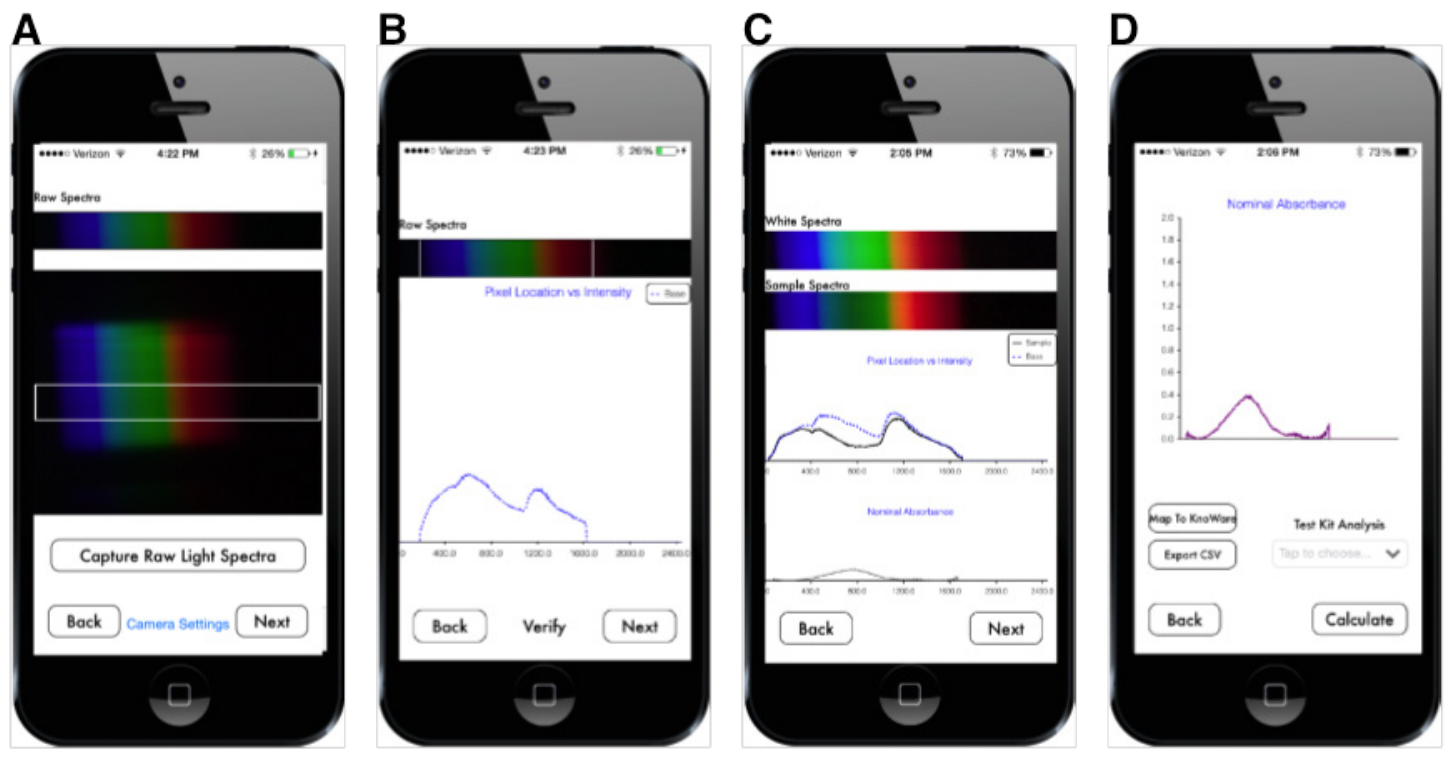

Figure 7.
A. Capturing a raw light spectrum.
B. RGB values for each pixel across the raw light spectrum converted to nominal in- tensity vs. pixel address plot.
C. Raw light spectrum from previous step (upper spectrum) compared with sample light spectrum (lower spectrum). Nominal intensity vs. pixel address for each spec- trum overlaid together.
D. Comparison of the raw and sample light intensity plots are represented as an ab- sorbance plot, which is characteristic for a given sample color. On-screen button can export data to KnoWare.

We conducted a series of measurements for standard nitrate solutions using our InSpector device and app and compared to those measured by a conventional spectrophotometer that costs over $\$ 20,000$. The comparisons of the determined absorbance spectra are shown in Figure 8A. Using these spectra, our InSpector app calculated the concentrations of nitrates using the ratios of the 2 peaks in the absorbance plots (one at $\sim 450 \mathrm{~nm}$ and one at $\sim 550 \mathrm{~nm}$, or $\sim 800$ and 1700 pixels in InSpector). While the overall ratios showed some error when comparing those from InSpector vs. the spectrometer (Figure 8B, y-value) the calibration curve was still linear, indicating that we can simply adjust the algorithm in the InSpector app to correct for this systematic error. In addition, we were able to reliably determine concentrations of nitrate below $2 \mathrm{ppm}$, which was better than using a color matching card (Figure 4). 
A

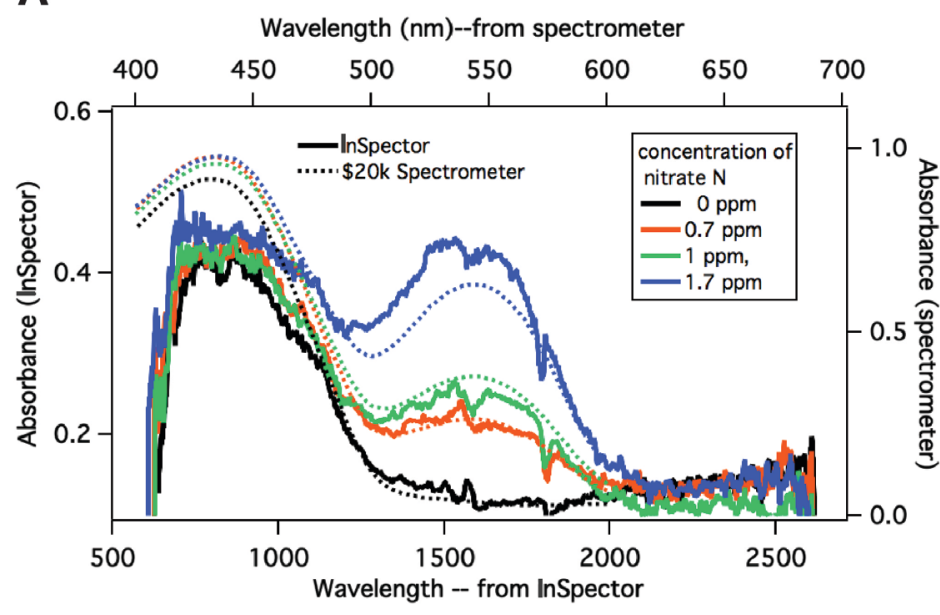

B

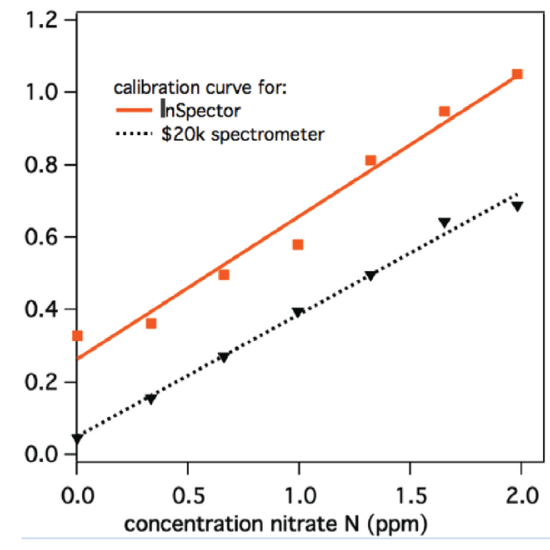

Figure 8.

A. Absorbance plot comparison using InSpector (solid lines) and a conventional \$20k spectrophotometer (dashed lines).

B. Calibration plots of the ratio of peak $2(\sim 550 \mathrm{~nm}$ or 1700 pixel\#) vs. peak $1(\sim 450 \mathrm{~nm}$ or 800 pixel\#) for different concentrations of nitrate $N$.

\section{Software Engineering}

The software engineering path of KnoWare is fairly unique. The idea was initially brought to a project-based service-learning software development class in the Department of Computer Science. Bringle and Hatcher (1995) define service-learning as a "course-based, credit bearing educational experience in which students (a) participate in an organized service activity that meets identified community needs, and (b) reflect on the service activity in such a way as to gain further understanding of curricular content, a broader appreciation of the discipline, and an enhanced sense of personal values and civic responsibility."

In this class, students were grouped into small software development teams of 6-8 students, and each team worked on a real-world software project with a real-client. Agile methodology, based on eXtreme Programming (Beck, 2000) and Scrum (Schwaber, 2004), was used by all teams to ensure efficient use of time and high levels of communication between themselves as well as the client. An iteration schedule developed by the instructor was used, that took advantage of Agile's focus on clients, as well as its iterative and incremental nature. The semester schedule was divided into five iterations 'I', I0-I4, each consisting of three weeks (see Table 1).

A team of students in the class began the initial development of the software package. Research guided the choice of appropriate software development tools and environments. The student teams operated with two-week iterations that involved high levels of interactions with the client to maintain consistent progress. At the end of each iteration, a useable version of the software was distributed for the client testing. Feedback from the client was used to modify the team activities during the subsequent iteration period. This agile process has been applied successfully in previous years by one of the authors, and was recommended by several other educators (Alfonso \& Botia, 2005; Chao \& Brown, 2009). 
Table 1: Software Project Semester Iteration schedule.

\begin{tabular}{ll}
\hline Week(s) & Service-learning project task \\
\hline $1 \& 2$ & Introduction to software engineering and the projects. \\
3 & $\begin{array}{l}\text { Formation of software development project teams and role assignments, as well as the software develop- } \\
\text { ment and technical communication combined teams. }\end{array}$ \\
4 & First customer meeting and Iteration $0\left(\mathrm{I}_{0}\right)$, which consisted of planning and a requirements analysis. \\
5 & Second customer meeting to review requirements and project plan. \\
$6 \& 7$ & Work on $\mathrm{I}_{1}$ and accompanying documentation. \\
8 & Deliver $\mathrm{I}_{1}$ (a working system) with accompanying release notes and updated project plan to client. \\
9 & Work on $\mathrm{I}_{2}$ and accompanying documentation. \\
10 & Deliver $\mathrm{I}_{2}$ (a working system) with accompanying release notes and updated project plan to client. \\
11 & Work on $\mathrm{I}_{3}$ and accompanying documentation. \\
12 & Deliver $\mathrm{I}_{3}$ (a working system) with accompanying release notes and updated project plan to client. \\
$13-15$ & Work on $\mathrm{I}_{4}$. Perform qualitative usability test. \\
16 & Deliver final software product and accompanying documentation (as a compiled help file) to client. \\
\hline
\end{tabular}

At the end of the semester, the project was passed on to a single graduate student for further development, while maintaining the Agile paradigm for strong communication and frequent updates with the client to ensure the product was developed as intended. The KnoWare and InSpector mobile applications were both written natively for iOS and Android (at the time of this manuscript writing, Android InSpector app is still in progress), while the web portal uses a standard web set up consisting of HTML, MySQL, PHP, JavaScript, and D3.js. All applications were developed to easily and intuitively interface in order to provide the user with the best experience possible. All software was written to be modular in nature to facilitate scalability and maintenance of the code. The iOS and Android mobile apps were written natively in Objective-C and Java respectively. This was done to ensure high in-app responsiveness, as well as to allow access to low-level hardware calls, which in the case of both the KnoWare and InSpector apps was critical given the dependence on various camera and GPS functions.

\section{Conclusion}

Increasing the prevalence and impact of citizen science efforts will benefit from facilitating the acquisition, sharing, and interpretation of data from large numbers of participants. The software and device technologies we have created are designed to create an adaptable platform that addresses these goals. The KnoWare app and web portal can be used to pose scientific queries to crowds of participants with flexible options for custom response types and meaningful color schemes. Participant responses are mapped in real time to a website for interpreting the results or sharing the raw data. In order to diversify the types of data that can be reliably generated by nonprofessional scientists, we also created InSpector, an app and inexpensive device that can be used to quantify the color of liquid solutions that are used in many different water quality test kits. The workflow created by these technologies reduces uncertainty in the data from subjective colormatching steps and streamlines data handling with real time logging and mapping of the geotagged data to a website for visualization.

Thanks to essential contributions of undergraduate and graduate students in software engineering and chemistry at our university, we have developed technologies designed to facilitate the participation of nonprofessional citizens in scientific research initiatives, and to increase the reliability 
and types of data that can be collected using mobile devices. Given the pressing concerns of harmful algal blooms that have affected many people in our region, we hope these products will benefit important research and public awareness objectives in the future.

\section{References}

Alfonso, M. I., \& Botia, A. (2005). An iterative and agile process model for teaching software engineering. In Proceedings of the 18th Conference on Software Engineering Education \& Training (CSEET'05) (pp. 9-16). Ottawa, Canada.

Beck, K. (2000). Extreme programming explained: Embrace change. Reading, Massachusetts: AddisonWesley.

Beierle, T. C. (2002). The quality of stakeholder-based decisions. Risk Analysis, 22(4), 739-749.

Bringle, R. G., \& Hatcher, J. A. (1995). A service-learning curriculum for faculty. Michigan Journal of Community Service Learning, 2, 112-122.

Cha, Y., \& Stow, C. A. (2015). Mining web-based data to assess public response to environmental events. Environmental Pollution, 198, 97-99.

Chao, J. T., \& Brown, J. K. (2009). Empowering students and the community through agile software development service-learning. Lecture Notes in Business Information Processing, 31 LNBIP, 104-113.

Cheung, M. Y., Liang, S., \& Lee, J. (2013). Toxin-producing cyanobacteria in freshwater: A review of the problems, impact on drinking water safety, and efforts for protecting public health. Journal of Microbiology, 5l(1), 1-10.

Conrad, C. C., \& Hilchey, K. G. (2011). A review of citizen science and community-based environmental monitoring: Issues and opportunities. Environmental Monitoring and Assessment, 176, 273-291.

Fischer, J., Dyball, R., Fazey, I., Gross, C., Dovers, S., Ehrlich, P. R., ... Borden, R. J. (2012). Human behavior and sustainability. Frontiers in Ecology and the Environment, 10(3), 153-160.

Fitzsimmons, E. G. (2014, August 3). Tap water ban for Toledo residents. The New York Times. Retrieved from http://www.nytimes.com/2014/08/04/us/toledo-faces-second-day-of-water-ban.html?_r=0

Gleeson, T., Wada, Y., Bierkens, M. F. P., \& van Beek, L. P. H. (2012). Water balance of global aquifers revealed by groundwater footprint. Nature, 488(7410), 197-200.

Gleick, P. H. (Ed.). (1993). Water in crisis a guide to the world's fresh water resources (1st ed.). New York, NY: Oxford University Press.

Gura, T. (2013). Citizen science: Amateur experts. Nature, 496, 259-261.

Heisler, J., Glibert, P. M., Burkholder, J. M., Anderson, D. M., Cochlan, W., Dennison, W. C., ... Suddleson, M. (2008). Eutrophication and harmful algal blooms: A scientific consensus. Harmful Algae, 8(1), 3-13.

Henry, T. (2014). Marcy Kaptur calls for a citizen-sampling brigade for Lake Erie water. The Toledo Blade. Retrieved from http://www.toledoblade.com/local/2014/09/15/Marcy-Kaptur-calls-for-a-citizensampling-brigade-for-Lake-Erie-water.html

Michalak, A. M., Anderson, E. J., Beletsky, D., Boland, S., Bosch, N. S., Bridgeman, T. B., ... Zagorski, M. A. (2013). Record-setting algal bloom in Lake Erie caused by agricultural and meteorological trends consistent with expected future conditions. Proceedings of the National Academy of Sciences of the United States of America, 110, 6448-6452.

Pahl-Wostl, C. (2002). Towards sustainability in the water sector - The importance of human actors and processes of social learning. Aquatic Sciences, 64(4), 394-411. 
Pollock, R. M., \& Whitelaw, G. S. (2005). Community-based monitoring in support of local sustainability. Local Environment, 10(3), 211-228.

Public Lab Staff. (2015). Foldable mini-spectrometer. Retrieved December 4, 2015, from https://publiclab.org/wiki/foldable-spec

Scheeline, A., \& Kelley, K. (2009). Cell phone spectrometer : Learning spectrophotometry by building and characterizing an instrument. Retrieved from http://www.asdlib.org/onlineArticles/elabware/Scheeline_Kelly_Spectrophotometer/Cell Phone Spectrometer Paper.pdf

Schwaber, K. (2004). Agile project management with Scrum. Redmond, Washington: Microsoft Press, A division of Microsoft Corporation.

Science Buddies Staff. (2015). See the light by making a cell phone spectrophotometer. Retrieved December 4, 2015, from http://www.sciencebuddies.org/science-fairprojects/project_ideas/Chem_p100.shtml\#summary

Smith, V. H., \& Schindler, D. W. (2009). Eutrophication science: Where do we go from here? Trends in Ecology and Evolution, 24(4), 201-207.

Vlek, C., \& Steg, L. (2007). Human behavior and environmental sustainability: Problems, driving forces, and research topics. Journal of Social Issues, 63(1), 1-19.

Vörösmarty, C. J., Green, P., Salisbury, J., \& Lammers, R. (2000). Global water resources: Vulnerability from climate change and population growth. Science, 289, 284-289.

Vörösmarty, C. J., McIntyre, P. B., Gessner, M. O., Dudgeon, D., Prusevich, A., Green, P., ... Davies, P. M. (2010). Global threats to human water security and river biodiversity. Nature, 467(7315), 555-561.

Wakabayashi, F., \& Hamada, K. (2006). A DVD spectroscope: A simple, high-resolution classroom spectroscope. Journal of Chemical Education, 83(1), 56-58.

Withers, P., Neal, C., Jarvie, H., \& Doody, D. (2014). Agriculture and eutrophication: Where do we go from here? Sustainability, 6(9), 5853-5875.

World Water Assessment Programme. (2009). The United Nations World Water Development Report 3: Water in a changing world. Paris, France: United Nations Educational, Scientific and Cultural Organization (UNESCO). Retrieved from http://www.unesco.org/new/en/naturalsciences/environment/water/wwap/wwdr/wwdr3-2009/downloads-wwdr3/

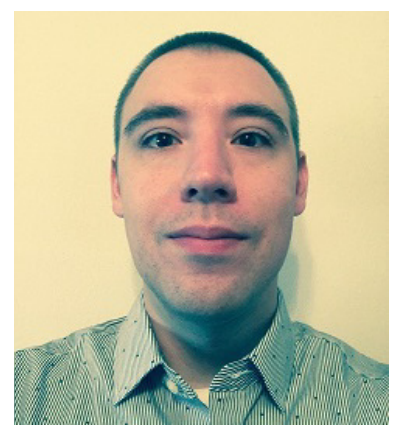

\section{Biographies}

Jeremy Storer is a Computer Science graduate student at Bowling Green State University. He received his B.Sc. in Computer Science from Bowling Green State University as well. Jeremy has a broad range of research interests consisting of Computational Intelligence, Machine Learning, Data Mining, High Performance Computing, and Data Visualization. 


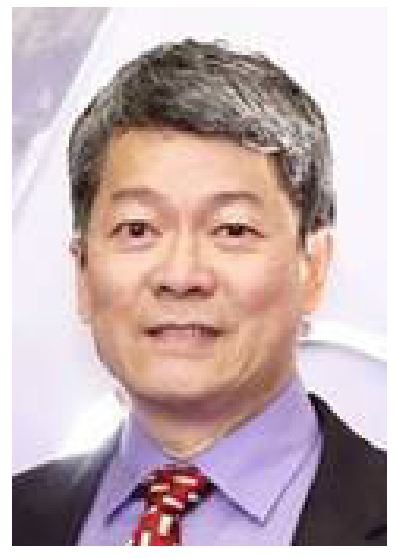

Dr. Joseph T. Chao is an Associate Professor and Chair of the Department of Computer Science at Bowling Green State University. He founded the Agile Software Factory at Bowling Green State University in 2008 with a grant from the Agile Alliance, which provides students with service-learning opportunities and real-world project experience in software engineering. Prior to entering academia, Dr. Chao gained more than seven years of industry experience working as Software Engineer, System Analyst, Software Architect, Project Manager as well as Director of Software Development. His research focus is on software engineering with special interests in agile software development, database systems, web and mobile technologies, and object-oriented analysis and design. Dr. Chao holds an M.S. in Operations Research from Case Western Reserve University and a Ph.D. in Industrial and Systems Engineering from The Ohio State University.

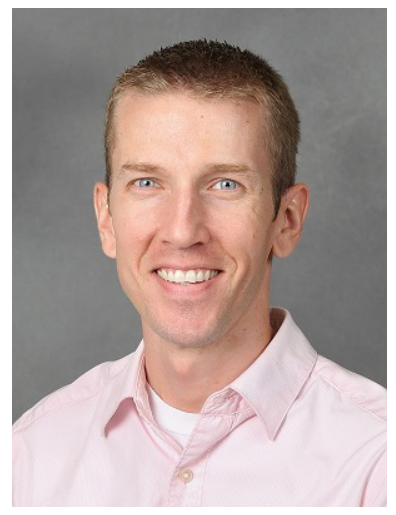

Dr. Andrew Torelli is an Assistant Professor of Chemistry at Bowling Green State University and brings broad experience at the interface of chemistry, the environment, software, and citizen engagement. Dr. Torelli teaches various courses related to biochemistry, and leads a research lab focused on relationship between the molecular structure and function of bacterial proteins. In addition, he has organized and participated in numerous science outreach experiences at the local, regional and national levels for various age groups working with contacts across industry, academia, and non-governmental organizations. Dr. Torelli received a B.Sc. degree from the State University of New York at Geneseo majoring in Biology and Computer Science, and a Ph.D. in Biophysics \& Structural Biology from the University of Rochester.

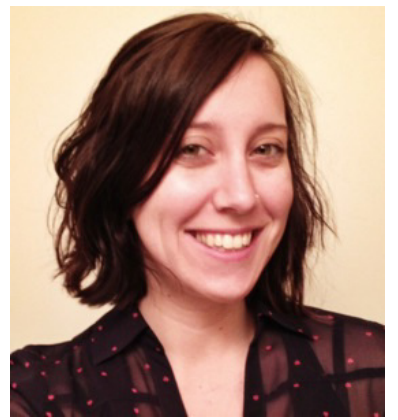

Dr. Alexis D. Ostrowski is an Assistant Professor of Chemistry at Bowling Green State University. She received a B.A. degree in Chemistry from Occidental College in Los Angeles, California and her Ph.D. in Chemistry from the University of California, Santa Barbara. Dr. Ostrowski received an NSF-IGERT fellowship at UCSB, and following her $\mathrm{PhD}$, was a NIH postdoctoral fellow at The Molecular Foundry, Lawrence Berkeley National Lab. She has experience in teaching and training students in high-level research in materials science, spectroscopy and photochemistry. Her group's research focuses on developing photo-responsive materials that utilize metal coordination. In addition, Dr. Ostrowski has participated in many different science education programs for students from K12 to college students, especially those focused on increasing participation in science by diverse groups. 